$24^{\text {th }}$ AIAA Aerodynamic Measurement Technology and Ground Testing Conference

28 June - 1 July, 2004, Portland, OR

\title{
Wall Interference Study of the NTF Slotted Tunnel Using Bodies of Revolution Wall Signature Data
}

\author{
Venkit Iyer* \\ Analytical Services \& Materials, Inc., Hampton, VA 23666 \\ David D. Kuhl \\ University of Maryland, College Park, MD 20742 \\ and \\ Eric L. Walker \\ NASA Langley Research Center, Hampton, VA 23681
}

\begin{abstract}
This paper is a description of the analysis of blockage corrections for bodies of revolution for the slotted-wall configuration of the National Transonic Facility (NTF) at the NASA Langley Research Center (LaRC). A wall correction method based on the measured wall signature is used. Test data from three different-sized blockage bodies and four wall ventilation settings were analyzed at various Mach numbers and unit Reynolds numbers. The results indicate that with the proper selection of the boundary condition parameters, the wall correction method can predict blockage corrections consistent with the wall measurements for Mach numbers as high as $\mathbf{0 . 9 5}$.
\end{abstract}

\section{Nomenclature}

$a \quad=$ slot width

$C_{D}, C_{L}=$ drag, lift coefficients

$C_{M}=$ pitching moment coefficient

$C_{3}, C_{4}=$ coefficients in the slotted wall boundary condition

$h=$ NTF tunnel width or height, $8.2 \mathrm{ft}$.

$k, k^{*}=$ slot parameters, non-dimensional or dimensional

$l=$ slot center-to-center distance (or slat width)

$M \quad=$ uncorrected Mach number

$Q \quad=$ uncorrected dynamic pressure

$V, V_{\text {ref }}=$ local wall velocity, tunnel reference velocity

$X, Y, Z=$ tunnel coordinates

$\alpha \quad=$ angle of attack

$\gamma \quad=$ ratio of specific heats

$\varepsilon=$ blockage parameter

$\Delta x=$ wall correction applied to quantity $x$

$\Phi \quad=$ perturbation potential

Subscripts:

$x=$ streamwise gradient

$n \quad=$ flow normal gradient

\section{Introduction}

THE National Transonic Facility (NTF) at the NASA Langley Research Center (NASA LaRC) is a highReynolds number transonic facility with a $25 \mathrm{ft}$. long test section with a $8.2 \mathrm{ft}$. square cross section. The facility

\footnotetext{
* Senior Engineer, ROME Group; Senior member, AIAA

${ }^{\dagger}$ Graduate student; Member, AIAA.

${ }^{\star}$ Research Engineer, Configuration Aerodynamics Branch, MS 286, Student member, AIAA.
} 
can operate with air or cryogenic nitrogen as the medium under pressure to achieve Reynolds numbers on the model approaching flight values. In order to achieve transonic operation and to alleviate wall effects, six longitudinal slots are provided on the floor and ceiling walls of the facility. The slots allow for streamline divergence into a large plenum chamber which is passively vented into the downstream part of the test section, just ahead of the diffuser using reentry flaps (see Fig. 1). The slots can also be sealed to reduce or fully block crossflow into the plenum using inserts, thus varying the tunnel ventilation or openness ratios from a maximum of $6 \%$ down to $0 \%$ (solid walls).

The subject of the present study is the estimation of wall interference at the NTF at varying openness ratios, Mach numbers and model sizes. A wall interference correction system based on the wall signature method (proposed by Hackett ${ }^{1}$ and developed by Ulbrich ${ }^{2}$ ) was implemented at the NTF in the solid-wall configuration in $1999^{3}$. The method has presently been extended to slotted-tunnel walls using a modified version of the wall interference code called TWICS ${ }^{4}$. As a part of code validation efforts, a wind tunnel test was recently conducted at the NTF with the main objective of studying blockage variation for different-sized models at different degrees of wall ventilation. In this test (NTF Test 142), three similar bodies of revolution models designated as C-4, C-3 and C-2 (scaled down in size in this order, see Fig. 2) were tested in the tunnel. This test was preceded by a calibration test (NTF Test 138) in which a pitot-static pressure probe (hereafter referred to as a $Q$-probe) was positioned at the model reference station centerline of the empty tunnel. In both tests, test data corresponding to $6 \%$ open test section (TS) with all six slots on top and bottom wall open, 4\% open TS (slot numbers 1, 2, 5 and 6 open on top and bottom walls) and 2\% open TS (slots 2 and 5 open) were obtained. Data were also obtained with the solid-wall boundary condition corresponding to all slots closed.

For each model, a series of Mach number data points were obtained at unit Reynolds numbers in the range 4 to 8 million/ft. Mach numbers ranged from 0.4 to near-sonic and higher depending on the model and configuration. Static pressures at an upstream location (Station -2, 15 feet ahead of model reference Station 13 for solid-wall cases, or in the plenum for 2, 4 and 6\% open cases) were measured and used in conjunction with the $Q$-probe static pressure measurements of Test 138 to arrive at the true reference Mach number at the model reference location of $X=13, Y=Z=0$. Wall pressure measurements were made at the available wall ports. The resulting data were preprocessed for data quality and input to the wall correction code TWICS.

An overview of the test plan for wall correction accuracy assessment is presented in Ref. 5. Free-air conditions (i.e., flow not limited by walls) are obtained by the addition of wall corrections to the measured data. Theoretically, the corrected data for different wall ventilations should be identical, which provides a means to validate the method. The corrected drag vs. Mach number is, for example, a good measure for validation. Due to the large volume of data and analysis performed, this paper will present the C-4, C-3 and C-2 body wall correction results only at various wall ventilation conditions. Details of TWICS code validation based on the drag-rise Mach number will be covered in a future paper.

\section{The NTF Wall Correction Method}

\section{A. General}

The effect of the wind tunnel walls on the measured parameters is quantified using the blockage parameter $\varepsilon$ and the angle of attack correction $\Delta \alpha$. The blockage parameter is the average of the streamwise perturbation velocity along the model centerline. It consists of the model volume blockage and the model wake blockage and represents a change (usually an increase) in the effective velocity around the model due to wall-induced streamline displacement. The change in angle of attack arises for a lifting body due to wall-induced perturbation velocity in the lifting direction and also due to a change in streamline curvature. The blockage parameter leads to corrections on $M$ and $Q$ given by

$$
\begin{aligned}
& \frac{\Delta M}{M}=\left(1+\frac{\gamma-1}{2} M^{2}\right) \varepsilon \\
& \frac{\Delta Q}{Q}=\left(2-M^{2}\right) \varepsilon
\end{aligned}
$$

$\Delta M$ and $\Delta Q$ are added to $M$ and $Q$ to get the corresponding free-air values. $\Delta \alpha$ is added to $\alpha$ to get the effective angle of attack. Further, $\Delta \alpha$ and $\Delta Q$ lead to corrections in $C_{D}, C_{L}$ and $C_{M}$. Gradient in blockage produces a buoyancy correction applied to $C_{D}$.

\section{B. The TWICS Method}


The Transonic Wall Interference Correction System ${ }^{4}$ (or TWICS) is a correction code based on the wall signature method developed for transonic tunnels with ventilated walls, originally for the NASA Ames 11-Ft Tunnel. It has been implemented for the NTF tunnel in the solid-wall as well as slots-open configuration. TWICS and its predecessor code WICS were developed by Ulbrich ${ }^{2}$ by using a strategy of globally fitting the wall signature.

TWICS uses the pressure signature at the walls (defined as the incremental value relative to tunnel empty signature) as the basis for computing wall interference corrections. The model is represented by a number of point doublets to simulate volume and wake blockage, and line doublets to simulate effects due to lift (in the general case - not used in this study). The far-field effect due to the assumed singularity distribution is matched with the wall signature. This is done in a global fitting procedure, which yields the strengths of the singularities as the solution. The perturbation velocities are then computed based on superposition of standard solutions of point and line doublets which are contained in pre-computed databases of perturbation velocity solutions. Corrections for each test point are obtained by interpolation from the database. Compressibility is modeled using Prandtl-Glauert scaling. As a result, there is an upper limit to the Mach number in the application of this method.

The TWICS code is designed to work in unison with a panel-method-generated database of wall interference solutions based on point doublets for blockage and line doublets for lift interference. By appropriately setting the boundary conditions in the panel method, walls with varying amounts of ventilation or openness can also be modeled. The point doublets are weighted in proportion to the cross section area of the test article. In TWICS, the effect of the sting is also modeled using a chain of weighted point doublets, in an analogous fashion to volume blockage. This shortcut implies the use of a considerably smaller tunnel calibration test matrix.

\section{Wall Correction Inputs}

\section{Wall signature}

The wall signature for a test point is defined in terms of the 12 rows with 30 orifices in each. In addition, the empty tunnel calibration data is required for a specified range of tunnel conditions. For full span models, this means the wall signature with the arc sector only in the tunnel. Input flags are used to de-select specific wall orifices that are not used in the calculation for a given test. The rejection criteria is based on wall data quality analysis.

\section{Tunnel reference}

Test point values of uncorrected force and moment values, Mach number, reference velocity at model center of rotation and a number of other test and model attitude parameters are required as inputs.

\section{Perturbation velocity database (PVD)}

This is a large table of pre-computed perturbation velocities used in signature matching and wall interference computation. The database depends on the wall orifices layout, tunnel section, Mach number and lift vector direction. For slots-open case using the homogeneous ventilated flow assumption, it also depends on the type of boundary condition set. The panel method code called ANTARES ${ }^{6}$ is used to generate these solutions.

\section{Model singularity distribution and geometry data}

This specifies how the point doublets and line doublets are distributed and weighted. Wing and fuselage cross sections and geometry data are also required. In addition, reference lines along which weighted averages of interference are to be computed and planes along which local values of wall interference are to be computed are also specified.

\section{Calculation Steps Used in TWICS}

1. Processing of input test data

For each test point, the wall signature is read in. Subsequently, the corresponding tunnel empty signature is interpolated from the calibration database and subtracted to get the incremental or 'tared' wall signature.

2. Computation of the equivalent line doublet strength

This is obtained from the measured lift and model geometry parameters. The strength is then distributed along the span as per specified weights based on the estimated wing loading distribution.

3. Interpolation from the perturbation velocity database (PVD)

This is done to estimate the lifting effect part of the signature at each port. The lifting part of the signature is then subtracted from the 'tared' signature to get the blockage effect at each port.

4. Least squares fitting and interpolation from PVD

This calculates the strengths of the point doublets. The two unknowns computed here are the volume blockage strength and the wake blockage strength. This step represents the core of the calculation procedure.

5. Computation of corrections

This is achieved by the interpolation from PVD to compute wall interference at any point in the test section (within reference grid limits) by superposition of all singularities. Mean corrections are then calculated using 
weighted averaging. Force and moment coefficient corrections are then computed. The streamwise distribution of blockage is used to estimate the buoyancy correction.

The steps are repeated if iteration using corrected tunnel parameters is specified. Corrections are computed for each point in a polar independently. The primary mean correction due to blockage is applied as corrections on Mach number, $M$ and dynamic pressure, $Q$ (added to corresponding measured values). Corrections on $C_{L}, C_{D}$ and pitching moment coefficient $C_{M}$ are computed based on the primary mean corrections of blockage and angle of attack. In addition, model-induced buoyancy correction is also calculated and added to $\Delta C_{D}$. The method also computes local variations of interference corrections, which are useful in determining if the averaging assumption is truly representative of the interference field in the model region.

\section{Model and Wall Configurations}

\section{A. Blockage Models}

An outline of the blockage models and support assembly projected to the test section wall is shown in Fig. 2. Also shown in this figure is the $Q$-probe assembly from the calibration test (Test 138). It is clear from this figure that the $Q$-probe assembly has approximately the same area distribution as the support sting assembly for the blockage models. Hence subtraction of the $Q$-probe wall signature from the blockage model wall signature will result in an incremental wall signature which is a first-order approximation to the signature from the model alone. The three models C-4, C-3 and C-2 tested are nearly identically shaped, but scaled down (affine models). Wall interference study of scaled models is used to validate the calculation method, since all the cases should correct to the same free-air conditions.

The important geometry data for the C-4, C-3 and C-2 bodies are given in the table below.

\begin{tabular}{|l|l|l|l|}
\hline & \multicolumn{1}{|c|}{ C-4 } & \multicolumn{1}{c|}{ C-3 } & \multicolumn{1}{c|}{ C-2 } \\
\hline Reference area, $\mathrm{ft}^{2}$ & 0.28711 & 0.18696 & 0.093482 \\
\hline Length, $\mathrm{ft}$ & 5.744 & 4.635 & 3.278 \\
\hline Leading edge $X, \mathrm{ft}$ & 10.266 & 10.266 & 10.266 \\
\hline Trailing edge $X, \mathrm{ft}$ & 16.01 & 14.901 & 13.544 \\
\hline Max dia, $\mathrm{ft}$ & 0.30231 & 0.24395 & 0.1725 \\
\hline Base area, $\mathrm{ft}^{2}$ & 0.0192 & 0.0125 & 0.008563 \\
\hline Volume, $\mathrm{ft}^{3}$ & 1.1497 & 0.6041 & 0.2136 \\
\hline
\end{tabular}

Table 1. Geometry data for the C-4, C-3 and C-2 models.

Note from Fig. 2 that the maximum cross section of the C-4 model is at $X=13$, whereas the maximum cross section locations for the $\mathrm{C}-3$ and $\mathrm{C}-2$ bodies are shifted forward relative to $X=13$. Note also the differences in the sting configurations.

The tunnel calibration data with the $Q$-probe assembly in the tunnel was obtained for the same Test 142 test parameters. A calibration database for each wall port is prepared from this data.

\section{B. Wall Signature Inputs}

As shown in Fig. 3, there are eighteen full or partial rows in the four tunnel walls with a total of 401 wall port measurements in the range $0<\mathrm{X}<19$ (limited thus to avoid the downstream region near the reentry flaps). Since TWICS currently has a limitation of using only 12 rows, the input pressures actually used in the TWICS calculation amounts to only about 260 or fewer ports. A number of different row combinations can be used in TWICS, which are specified by way of a port selection file. An example of port selections is shown in Figure 3, consisting of 3 rows (the center row and the two adjacent rows on either side of it) from each wall. The numbering of the rows used in the tunnel instrumentation hookup sheet and in TWICS (numbered from 1 to 12) are also shown in these plots. The TWICS numbering is used to refer to the rows in all the figures in this paper.

\section{Singularity Distribution for TWICS}

The singularity distribution used corresponds to 20 point doublets arrayed along the body centerline and 35 point doublets spaced at $0.5 \mathrm{ft}$ starting from the tail of the body to capture the wake effect. The blockage doublets are weighted in proportion to the local cross-section area of the model. The wake doublets are equally weighted. It is 
not necessary to model the blockage due to the model support structure since it is effectively removed when the empty tunnel calibration data is subtracted.

\section{Perturbation Velocity Database}

Solution of the wall interference flow field for a flow singularity placed at a particular location in the tunnel using the potential flow approximation with prescribed boundary conditions is accomplished using the panel method ANTARES ${ }^{6}$. A database of solutions is generated by placing point doublet singularities along the line $7<X<33$, $Y=Z=0$ with a $\Delta X$ of $1.0 \mathrm{ft}$. As recommended in the panel code, the $X$ value of $33 \mathrm{ft}$ for the most downstream panels is approximately 3 times the average diameter of the tunnel in relation to the $X=13$ point. The wall signature at all the wall port locations is calculated as well as the interference velocities along the centerline $0<X<26$ with a $\Delta X$ of 1 $\mathrm{ft}$. The calculations are repeated at the following 12 Mach numbers: 0.0, 0.3, 0.55, 0.7, 0.8, 0.85, 0.875, 0.9, 0.925, 0.94, 0.95 and 0.96. Results presented are for Mach numbers below 0.95 only.

For the ventilated cases, the homogeneous slotted-wall boundary condition is used with the floor and ceiling walls assumed to be at a constant $6 \%, 4 \%$, or $2 \%$ openness and $0 \%$ openness (i.e., no crossflow) at the two sidewalls. Perturbation velocity databases for the $0 \%$ open case are additionally calculated using the Method of Images (MOI) program for verification.

The flow field in the vicinity of the slots is a complicated problem dependent on the model, the tunnel conditions, the slot geometry, the plenum volume and the flow through the reentry flaps. Since it is impractical to implement a detailed model of the slot flow, an approximate boundary condition based on a homogeneous ideal slotted-wall is used. In this approach, the boundary condition at the wall is specified as

$$
\phi_{x}+C_{3} \phi_{n}+C_{4} \phi_{x n}=0
$$

The value of $c_{4}$ is obtained empirically as a multiple of the non-dimensional slot flow factor $k$ defined as

$$
k=\frac{l}{\pi} \ln \left(\csc \frac{\pi a}{2 l}\right)
$$

For the NTF slotted wall configuration, the average slot width $a$ of 0.984 in. and a slot center-to-center distance $l$ of $1.366 \mathrm{ft}$ is used. With 6 slots open on the top and bottom walls, the ventilation percentage is $6 \%$ as given by $6 a / h$. Corresponding to this, $C_{4}$ is obtained as a dimensional value $C_{4}=k^{*}=F l k$ with $F$ as an empirical factor used to scale up the theoretical value of $k$ given by Davis and Moore ${ }^{8}$ as in Eq. (4). Based on work presented by Everhart in Ref. 7, the value of $F$ for the NTF is 4.75 , which results in a $k$ value of 3.6 or a $k^{*}$ value of $4.92 \mathrm{ft}$. For the $4 \%$ and $2 \%$ open cases, the values of $k^{*}$ are $8.6 \mathrm{ft}$. and $21.6 \mathrm{ft}$., respectively, corresponding to the increased slat widths.

$C_{3}$ is a coefficient that represents viscous flow through the slots. Due to lack of basic experimental slot studies to determine $C_{3}$, a strategy of selecting this coefficient based on the best fit with the measured wall signature is used. Use of the viscous slot flow term also means a corresponding change in the $C_{4}$ coefficient, which was originally based on inviscid slot flow. Details are given in the Results section.

\section{Results}

\section{A. C-4 Body in 0\% Open Tunnel}

Comparison of the incremental wall signatures measured in the tunnel and the corresponding values computed from TWICS gives a good indication of accuracy of the method. Fig. 4 shows representative wall signature comparisons for four Mach numbers $(M=0.4,0.7,0.9,0.93)$ for the $\mathrm{C}-4$ model in the $0 \%$ ventilation case. In this figure, due to reason of symmetry, specific rows are grouped together. Note that a good fit is obtained by TWICS for Mach numbers up to 0.93 . Since this is a solid-wall case, the wall boundary condition is precisely known and hence a good agreement is expected. Mach numbers $>0.93$ led to choking conditions in the tunnel which limited the applicability of TWICS. Also note the large increase in the wall signature for Mach numbers 0.9 and 0.93 compared to the lower Mach numbers. The measurements are seen to have the adequate resolution and repeatability required for the wall signature method. Calculations were repeated with the MOI perturbation velocity database and verified to reproduce the results.

The mean values of corrections for Mach number and $Q$ as well the blockage parameter $\varepsilon$ from selected test points for the C-4 body in the 0\%-open wall configuration are given in Fig. 5. The blockage increases with Mach number in an exponential manner with a maximum blockage of $2 \%$ at Mach 0.93 . The correction for Mach number is a maximum of 0.025 at Mach 0.93 . The maximum correction on $Q$ is about $3.5 \%$ of the uncorrected value for the lowest Reynolds number run. Hence the maximum correction for $C_{D}$ due to blockage is also about $3.5 \%$. The 
corrected $C_{D}$ will be smaller than the value obtained from balance measurements. The effect of Reynolds number in the range tested ( 4 to 8 million per $\mathrm{ft}$ ) appears to be minimal. Also note that results from repeat points are tightly clustered demonstrating the excellent repeatability of the measurements and hence the wall corrections.

The variation of the standard deviations of the TWICS fit to the measured wall signature as a function of the Mach number shows only a slow increase with Mach number for Mach numbers less than or equal to 0.93 . Above this value, the standard deviation increases significantly, again indicative of the choked flow in the tunnel. The standard deviation levels for $M<0.93$ are quite small compared to previous tests as is expected for this non-lifting symmetrical blockage model.

\section{B. C-4 Body in $6 \%$ Open Tunnel}

Fig. 6 shows the wall signature comparisons for four Mach number $(M=0.4,0.7,0.9,0.95)$ test points for the C-4 model in the $6 \%$ open tunnel with a $C_{4}$ (or $k^{*}$ ) value of 5 in the boundary condition (refer to Section V). Note that the Mach number limit of 0.95 is higher than the $0 \%$ case since tunnel is now ventilated. The plot scale used is $2 / 5^{\text {th }}$ of the scale used in Fig. 4 for the solid-wall case. It is noted that for $M>0.9$, the magnitude of the measured wall signature is only about $1 / 5$ th of the $0 \%$ open case. For lower Mach numbers such as 0.4 , the wall signature is about one half the magnitude of the $0 \%$ open case.

It is obvious from the figure that the quality of fit from TWICS is not good. The location of the peak of the fit curve does not coincide with that of the data. Another notable difference is that the trough in the wall signature ahead of the body (which corresponds to decreased velocities) is not well-captured. Also note that, since this is a blockage-only case, the singularity strengths are scaled to fit the measurements; therefore the shape of the fitted curve is the only key factor.

Work by Everhart ${ }^{7}$ has shown that viscous flow in the slots is an important effect which results in the shifting of the peak wall signature in the model region. This corresponds to making changes to the $C_{3}$ and $C_{4}$ coefficients in Eq. (3). Due to lack of precise knowledge of these coefficients, different combinations as shown in the table below were tried.

\begin{tabular}{|l|l|l|l|l|l|l|}
\hline$C_{3}$ & 0 & 1 & 2 & 1 & 2 & 0 \\
\hline$C_{4}$ & 5 & 5 & 5 & 2 & 2 & 9 \\
\hline
\end{tabular}

Table 2. Values of boundary condition coefficients used in ANTARES.

Corresponding perturbation velocity databases for all the Mach numbers were computed and wall corrections recalculated. The addition of viscous effects improves the wall signature fit considerably. The best wall signature fit obtained was for the $C_{3}=C_{4}=2$ case, shown in Fig. 7. There is a better agreement in the location of the peak and the upstream trough of the wall signature across the Mach number range compared to Fig. 6 with $C_{4}=5 ; C_{3}=0$.

Fig. 8 shows the blockage corrections resulting from the different boundary condition coefficients. Overall, the corrections are almost an order of magnitude smaller compared to the solid-wall case in the higher end of Mach number range. The blockage results from the $C_{3}=C_{4}=2$ case are lower compared to the non-viscous boundary condition, especially at the higher end of the Mach number range. The standard deviation of the TWICS fit relative to the measured values is also the lowest for the $C_{3}=C_{4}=2$ case.

\section{Comparison of Results for $6 \%, 4 \%, 2 \%$ and $0 \%$ Wall Ventilation}

Based on the results from the previous section, boundary condition coefficients for the $4 \%$ and $2 \%$ open cases were taken to be as follows. $C_{3}=2 ; C_{4}$ values scaled up to 3.5 and 8.8 for the $4 \%$ open and $2 \%$ open cases respectively, from the value of 2 . Fig. 9 shows an example of the comparison between measured and predicted incremental wall velocities at Mach 0.95 for the C-4 body in a 2\%-open test section. The variation of blockage factors for the C-4 body with percentage ventilation is shown in Fig. 10. Even a 2\% open test section produces a substantial reduction in blockage. The exponential increase of blockage with Mach number seen for the solid-wall case is considerably less for the ventilated cases. As also shown in Fig. 10, the global standard deviation of the TWICS fit to the measurements stays at about the same level irrespective of the ventilation percentage. As a result, wall correction accuracies can be expected to be at the same level for the different wall boundary conditions.

\section{Comparison of Results for C-4, C-3 and C-2 bodies}

The effect of body size on blockage for the 6\% open condition is shown in Fig. 11. The figure shows the expected decrease in blockage with size with a repeatable trend even for the relative small C-2 model. This demonstrates the sensitivity of the TWICS model to changes in model size. 


\section{E. Effect of Reynolds Number}

Change in unit Reynolds numbers did not produce any discernable trend in the blockage apparently due to the small range used ( 4 to 8 million per $\mathrm{ft}$ ). The changes were only of the same magnitude as the scatter in the computed blockage from various repeat test points.

\section{F. Comparison with Classical Results}

Fig. 12 summarizes the comparison of TWICS results with results obtained from a classical formulation for all the test points below Mach 0.95 (including all repeat points). The classical formulation uses the model average diameter, length and volume and the tunnel geometry and Mach number to first compute the closed-wall blockage value based on charts computed from a potential flow-based analysis (see Ref. 9, for example). The ventilated tunnel blockages are then computed by scaling the blockage based on homogeneous slot flow correlations developed by Pindzola and $\mathrm{Lo}^{10}$. Note that the classical method used here does not include any viscous effects.

Refer to the top left part of Fig. 12. The solid-wall blockages for all the three models appear to be generally consistent with the TWICS results. However, for Mach numbers > 0.8, the TWICS blockage values are slightly less than the classical results for all the three models. With ventilation however, the blockage values are significantly reduced (note the change in the Y scale). Note also that the TWICS results are significantly less than the classical results, especially at higher Mach numbers. Refer to bottom right part of Fig. 12 for the $6 \%$ open results. The TWICS blockage values are substantially lower than the classical results. This lack of agreement is attributable to the deficiency in the classical model which does not take into account the viscous flow in the slots, especially at higher Mach numbers. Since TWICS uses the actual wall signature, it does a better job of modeling the real flow in the tunnel. The $4 \%$ and $2 \%$ ventilation cases also show similar differences in blockage at the higher end of the Mach number range.

\section{Conclusion}

Implementation of the wall correction method TWICS for the slots-open and slots-closed configurations of the NTF has been described. Application to bodies of revolution models recently tested at the facility has been presented. The effects of model size and the ventilation percentage have been presented. Results obtained from classical methods are shown for comparison. The results from this study should prove useful in the calculation of wall corrections for lifting models at the NTF.

\section{Acknowledgments}

Support from NASA Langley Research Center under contract NAS1-00135 is gratefully acknowledged. The authors would like to thank Dr. J. L. Everhart and Dr. M. J. Hemsch for many fruitful discussions. Dr. N. Ulbrich provided the TWICS and ANTARES codes and the documentation used in this study.

\section{References}

${ }^{1}$ Hackett, J.E., Wilsden, D.J., and Lilley, D.E., "Estimation of Tunnel Blockage from Wall Pressure Signatures: a Review and Data Correlation," NASA CR-152241, March 1979.

${ }^{2}$ Ulbrich, N., "The Real-time Wall Interference Correction System of the NASA Ames 12-foot Pressure Tunnel," NASA CR1998-208537, July 1998.

${ }^{3}$ Iyer, V., Everhart, J., Bir, P., and Ulbrich, N., "Implementation of the WICS Wall Interference Correction System at the National Transonic Facility," AIAA 2000-2383, June 2000.

${ }^{4}$ Ulbrich, N. and Boone, A.R., "Determination of the Wall Boundary Condition of the NASA Ames 11ft Transonic Wind Tunnel," AIAA 2001-1112.

${ }^{5}$ Walker, E.L., "Measuring Wall Interference Correction Accuracy: An Overview of the NTF Program (Invited)," AIAA 20040770, January 2004.

${ }^{6}$ Ulbrich, N., "Description of Panel Method Code ANTARES," NASA CR-2000-209592.

${ }^{7}$ Ewald, B. F. R. (Editor), "Wind Tunnel Wall Correction,” AGARDograph 336, October 1998, pp. 5-24 to 5-32.

${ }^{8}$ Davis, D. and Moore, D., "Analytical Studies of Blockage- and Lift-Interference Corrections for Slotted Tunnels Obtained by the Substitution of an Equivalent Homogeneous Boundary for the Discrete Slots," NACA RM-L53-E07b, 1953.

${ }^{9}$ Barlow, J.B., Rae, Jr., W.H., Pope, A, Low-Speed Wind Tunnel Testing, Third Ed., John Wiley \& Sons, 1999.

${ }^{10}$ Pindzola, M., and Lo, C.F., "Boundary Interference at Subsonic Speeds in Wind Tunnels with Ventilated Walls," AEDC-TR69-47, May 1969. 


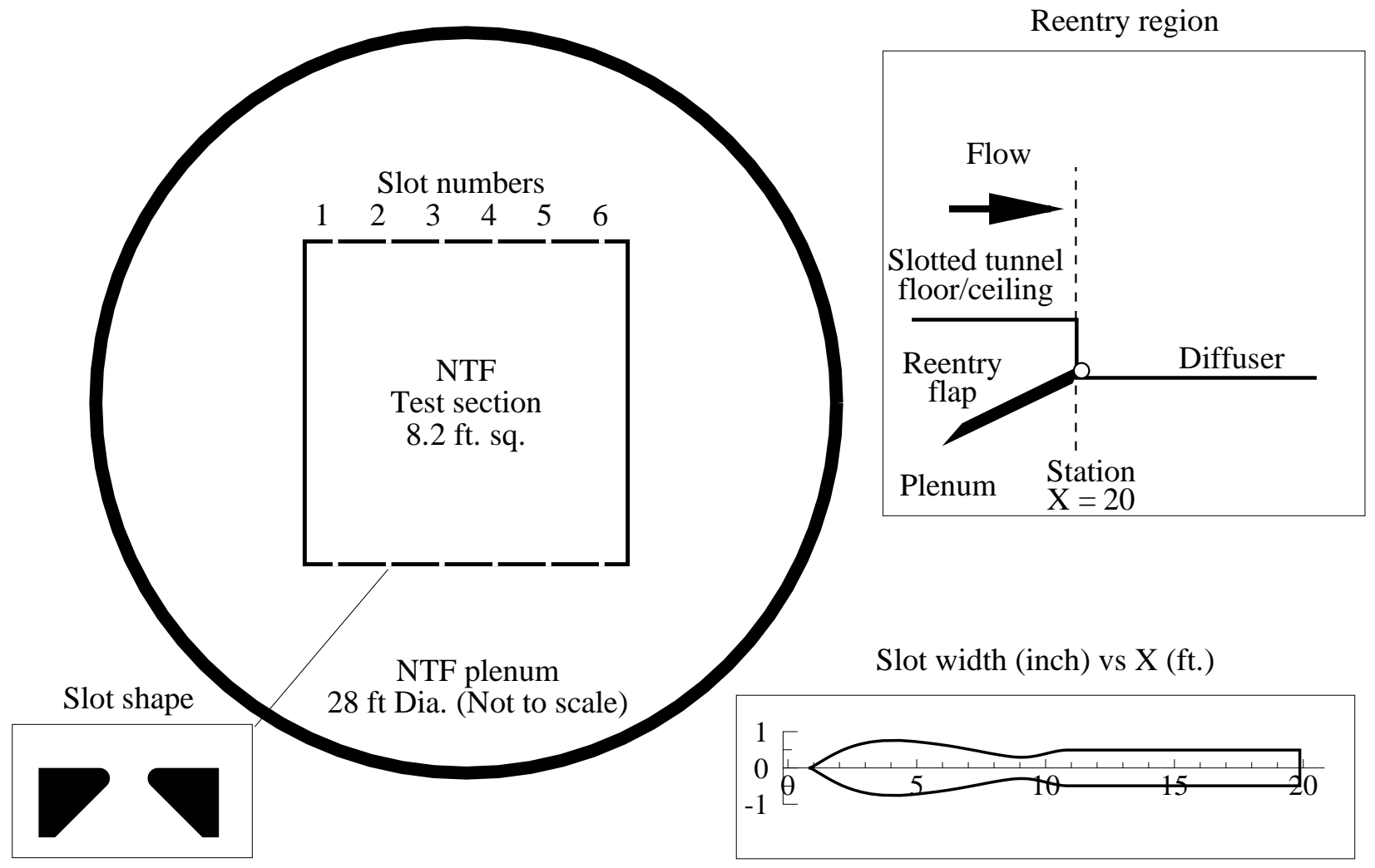

Figure 1. The NTF slotted-wall configuration.

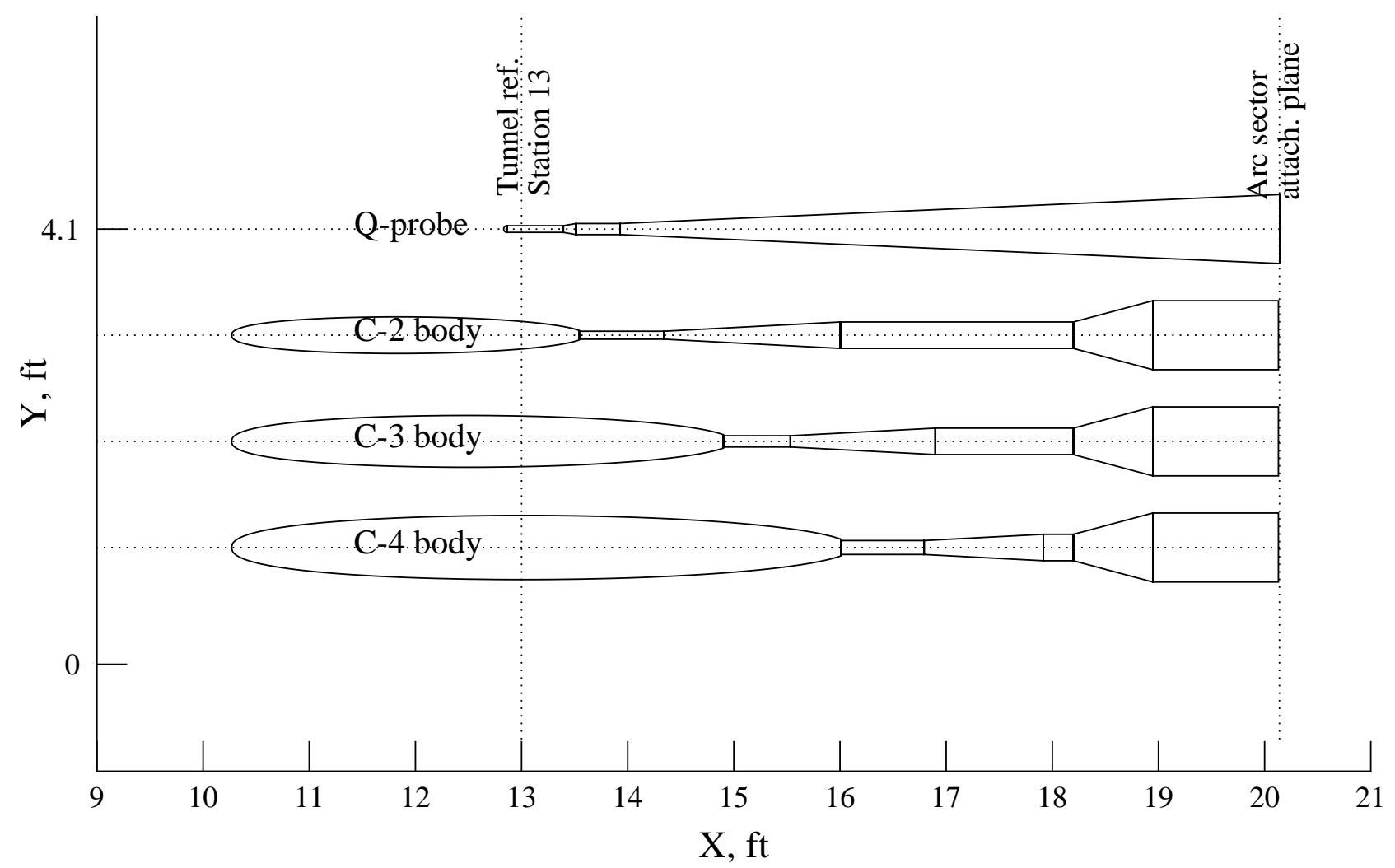

Figure 2. Comparison of model sizes and locations in tunnel coordinates; models are shifted in Y by 1, 2, or $3 \mathrm{ft}$. 

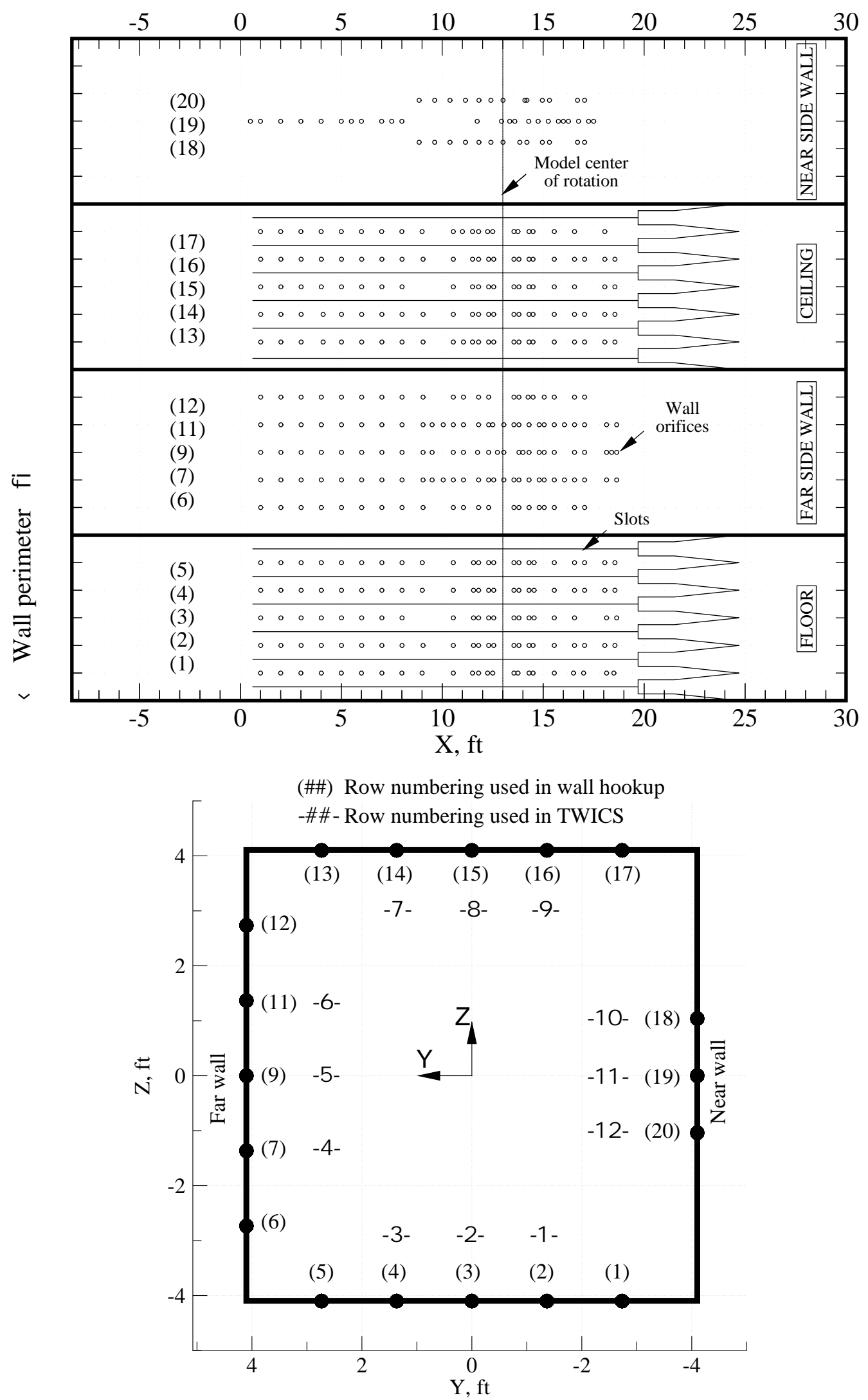

(View looking downstream)

Figure 3. NTF wall ports and row locations in the range $0<X<19$ 


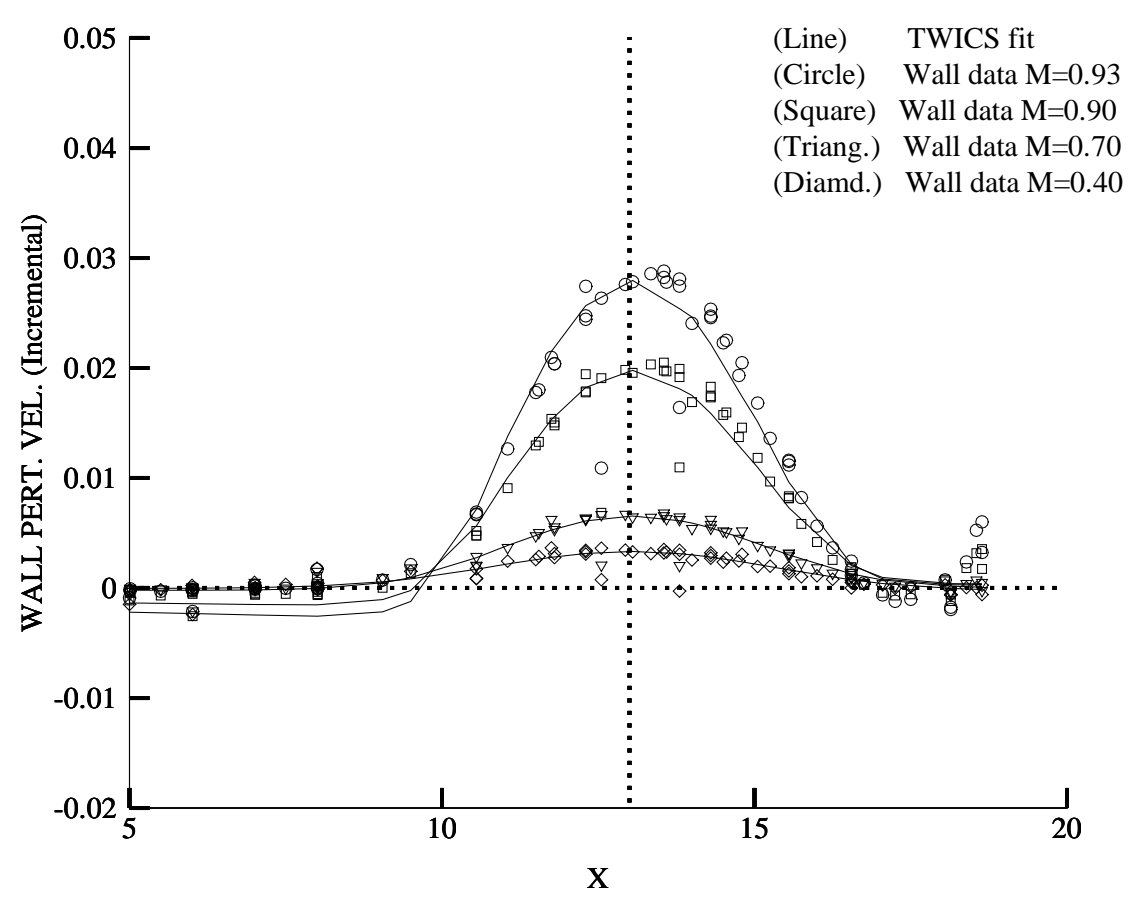

8 Off-centerline rows

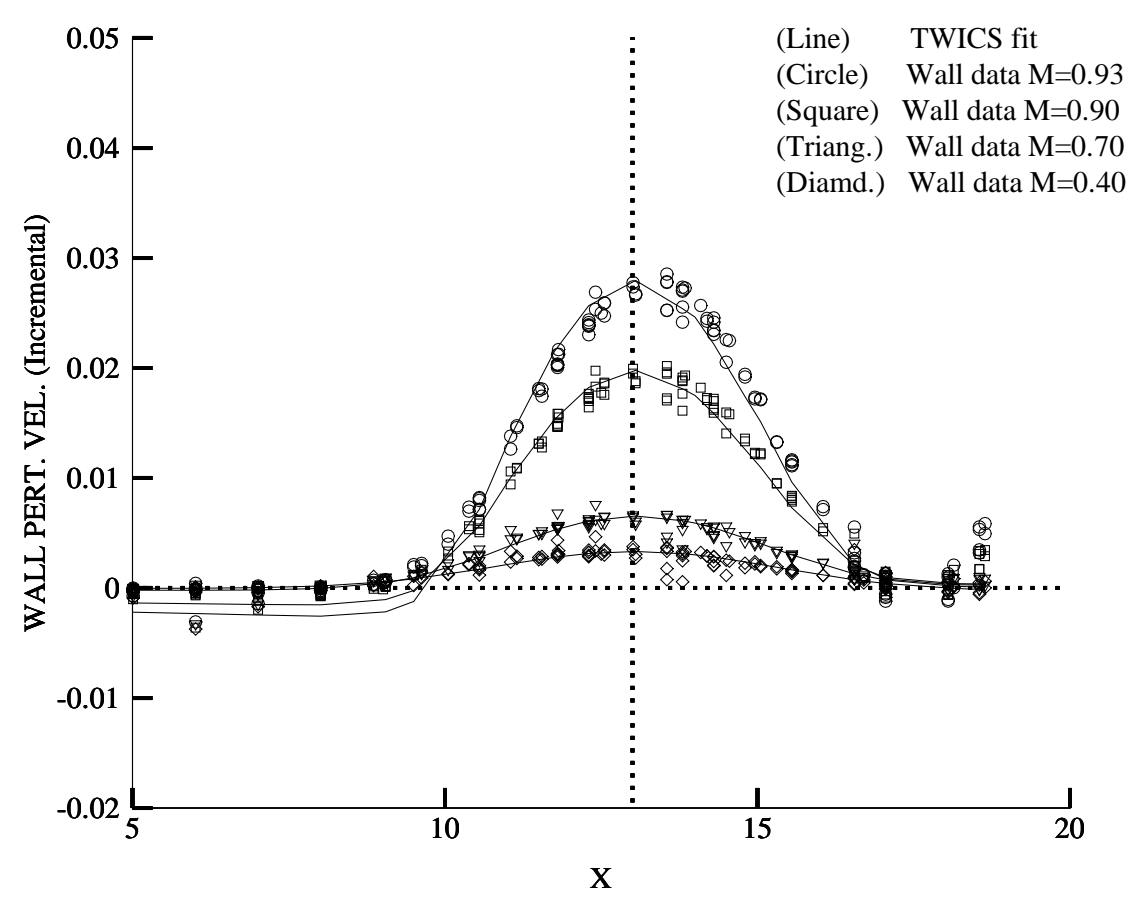

Figure 4. Measured wall signature and TWICS fit for the C-4 body in the NTF solid-wall configuration, $\mathrm{Re}=4.6 \mathrm{million} / \mathrm{ft}$. 

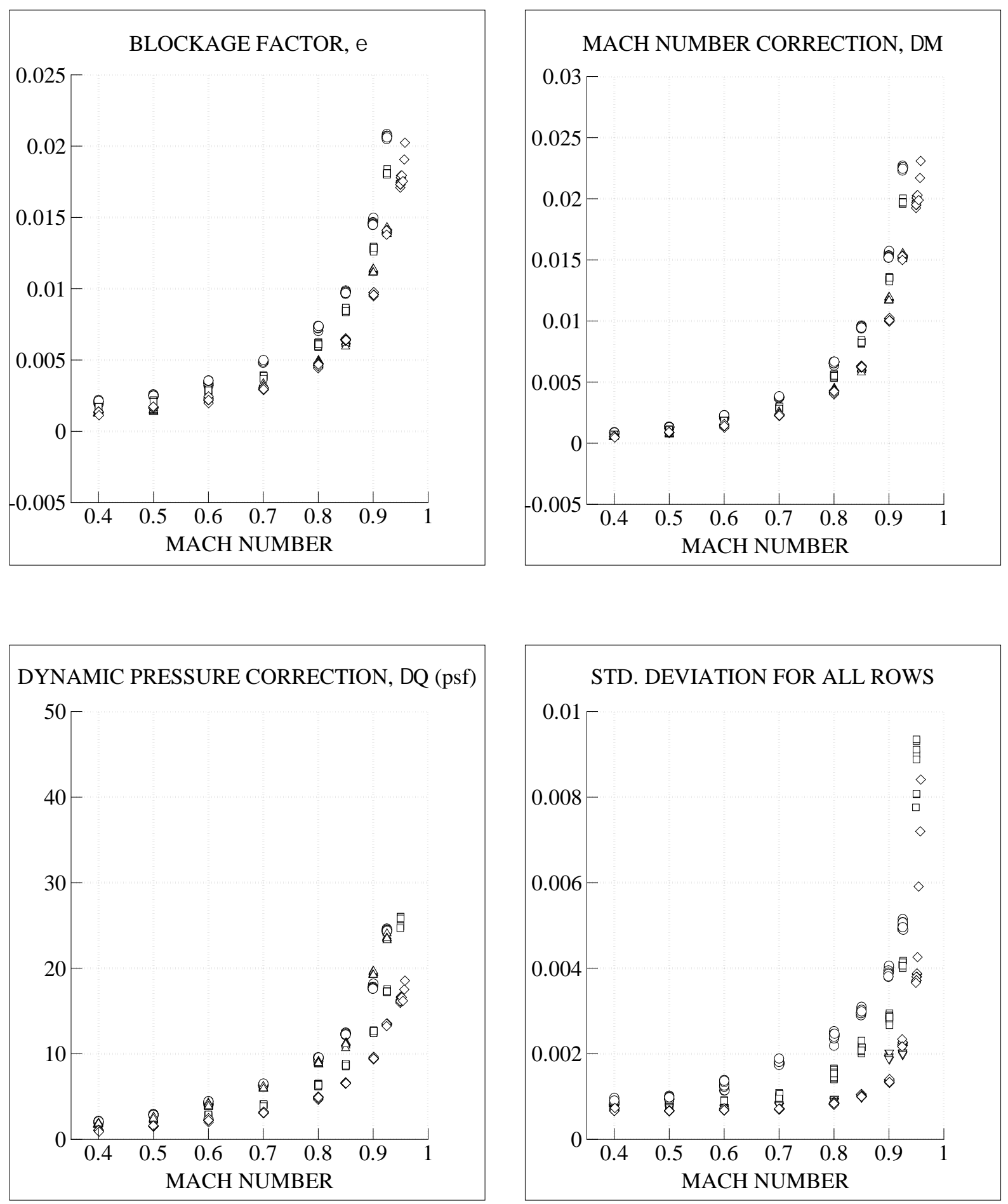

Figure 5. Wall interference corrections from TWICS, C-4 body in NTF solid-wall configuration Test points are: circle- $\mathrm{Re}=5.7$; square- $\mathrm{Re}=4.6$; triangle- $\mathrm{Re}=8.1$; diamond $\mathrm{Re}=4.6$ million $/ \mathrm{ft}$ Standard deviations of TWICS fit are in $\left(\mathrm{V} / \mathrm{V}_{\text {ref }}\right)$ with all 12 rows included in the computation. 
2 Centerline rows (floor and ceiling)

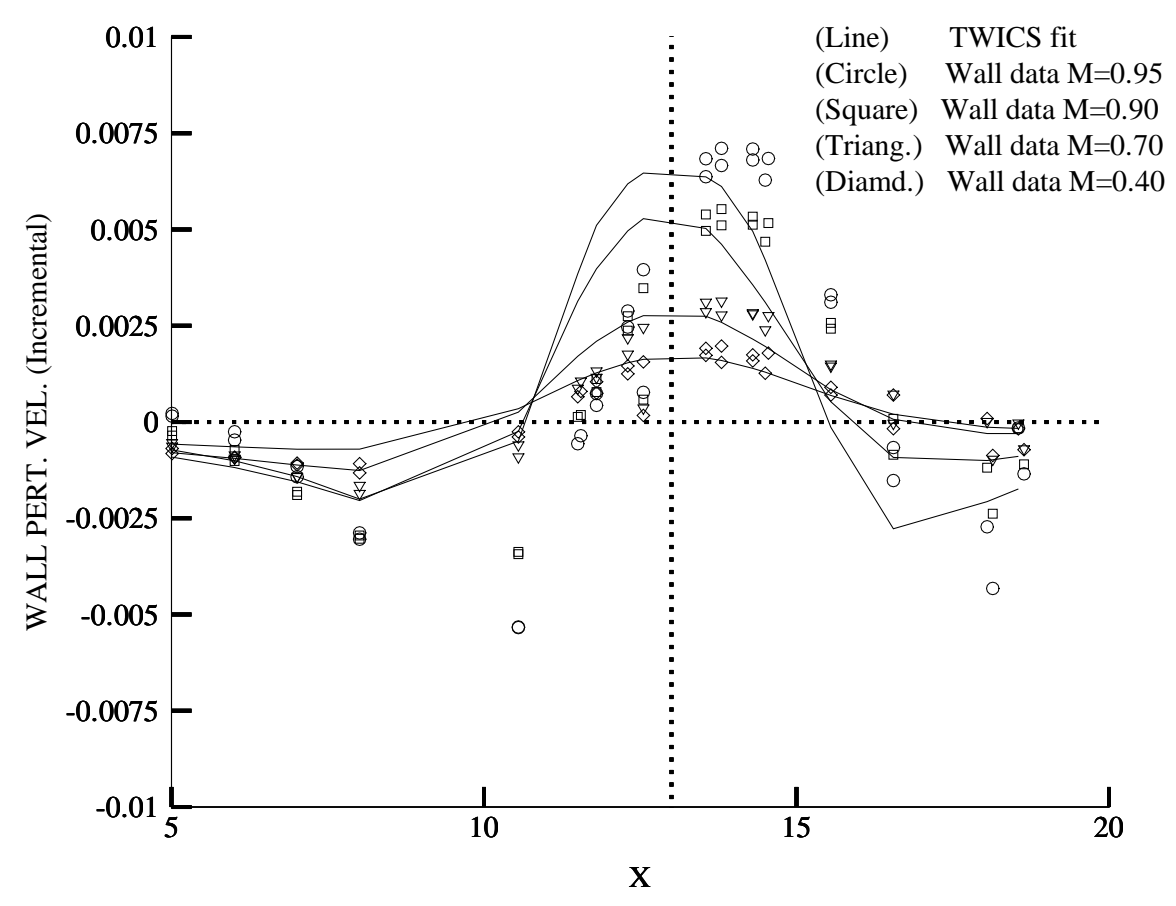

4 Off-centerline rows (floor and ceiling)

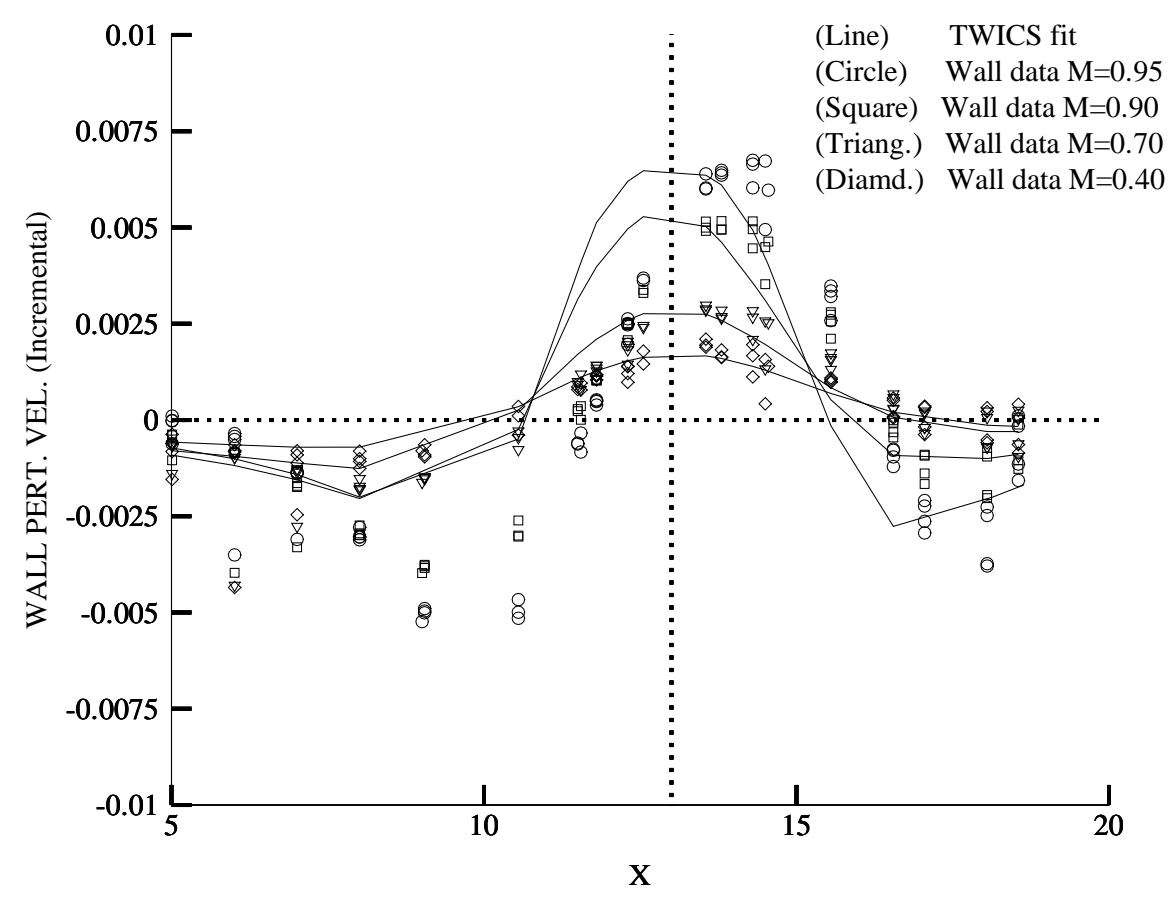

Figure 6. Measured wall signature and TWICS fit for the C-4 body in the NTF $6 \%$-open wall configuration, $\mathrm{Re}=4.6 \mathrm{million} / \mathrm{ft}$. 
2 Centerline rows (floor and ceiling)

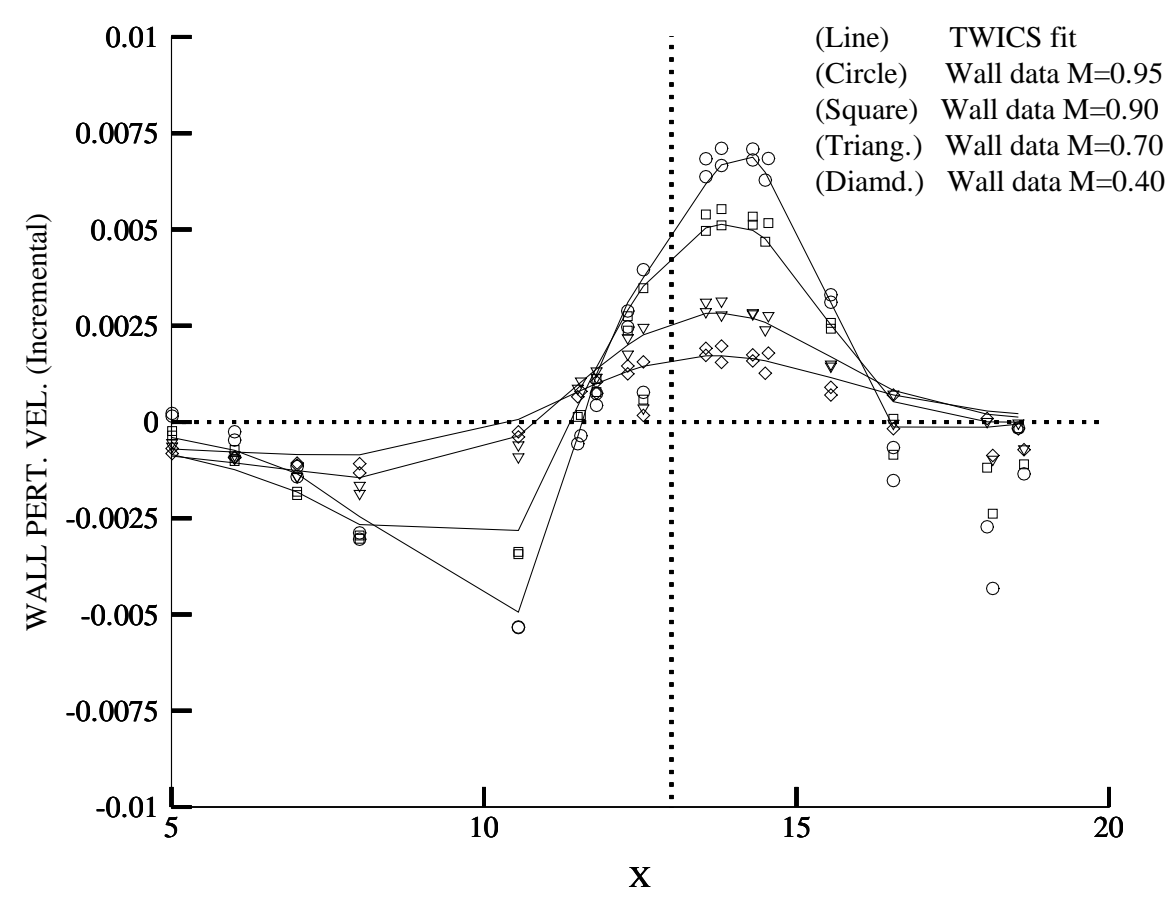

4 Off-centerline rows (floor and ceiling)

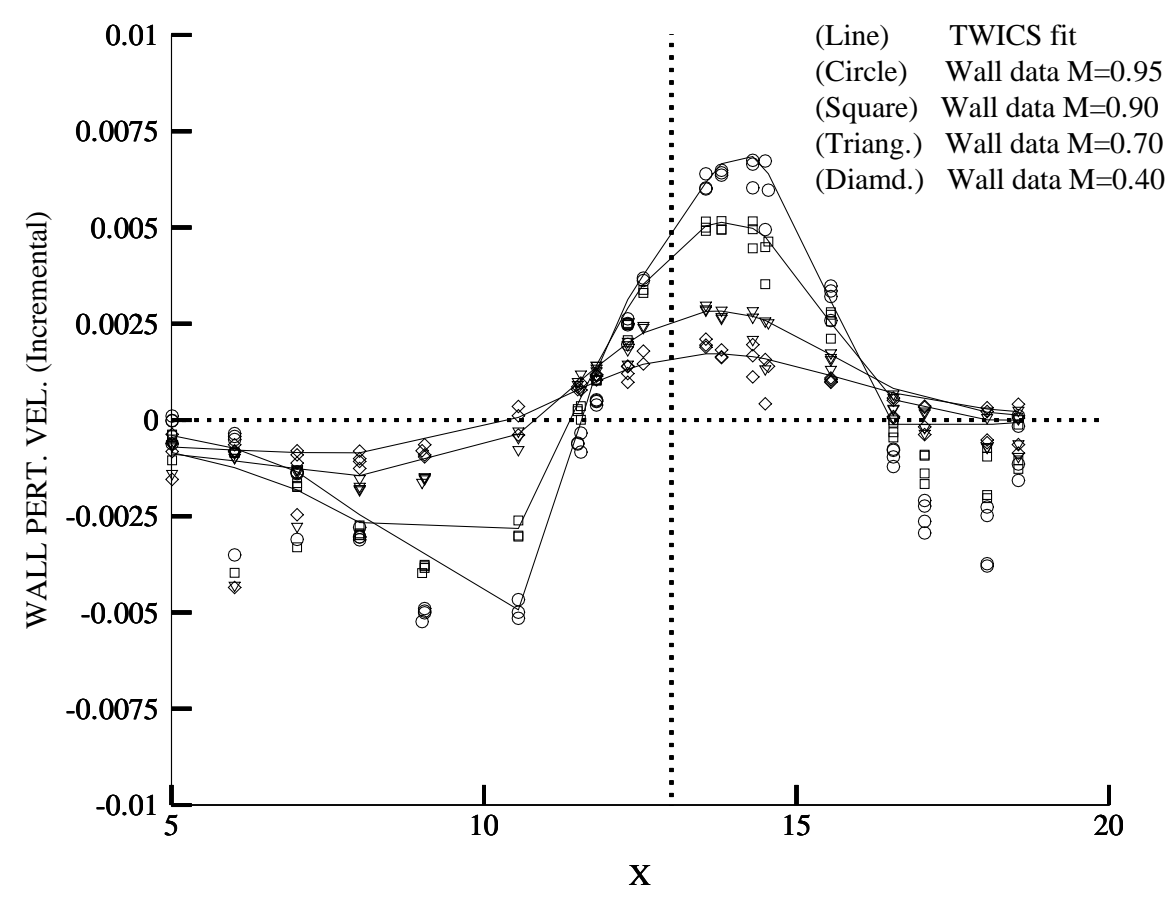

Figure 7. Measured wall signature and TWICS fit for the C-4 body in the NTF $6 \%$-open wall configuration with boundary condition coefficients $\mathrm{C}_{4}=2, \mathrm{C}_{3}=2$. 

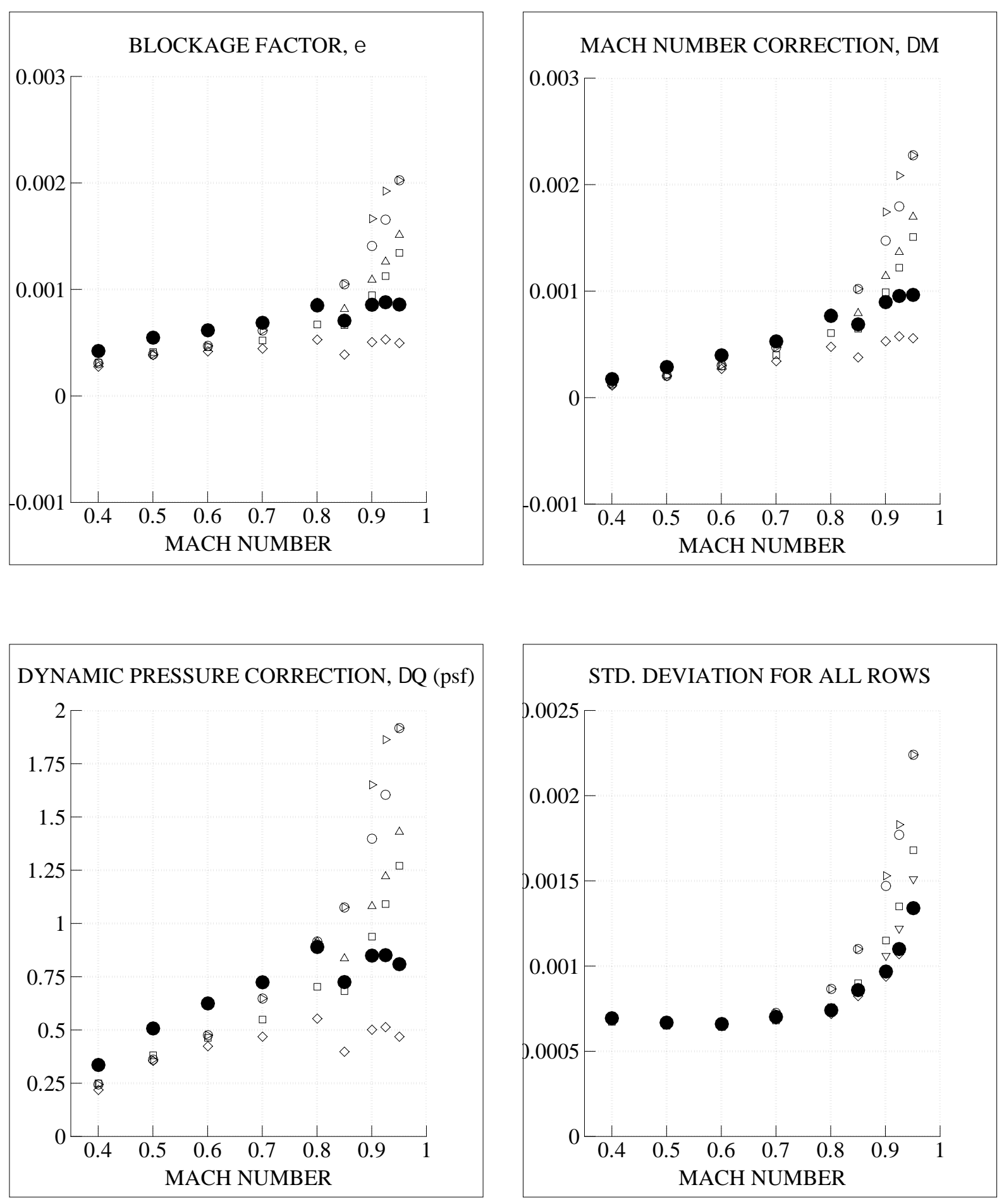

Figure 8. Wall interference corrections from TWICS, C-4 body in NTF 6\%-open wall configuration Cases are: circle- $C_{4}=5, C_{3}=0$; square- $C_{4}=5, C_{3}=1$; triangle- $C_{4}=5, C_{3}=2$; diamond- $C_{4}=2, C_{2}=1$; filled circle- $C_{4}=2, C_{3}=2$; right triangle- $C_{4}=9, C_{3}=0$. 
$\mathrm{X}$-axis: Streamwise distance in $\mathrm{ft}$; Y-axis: Incremental perturbation velocity at the walls Symbols: Measured perturbation velocity minus tunnel empty velocity

Lines: TWICS global fit of the measured incremental velocity.
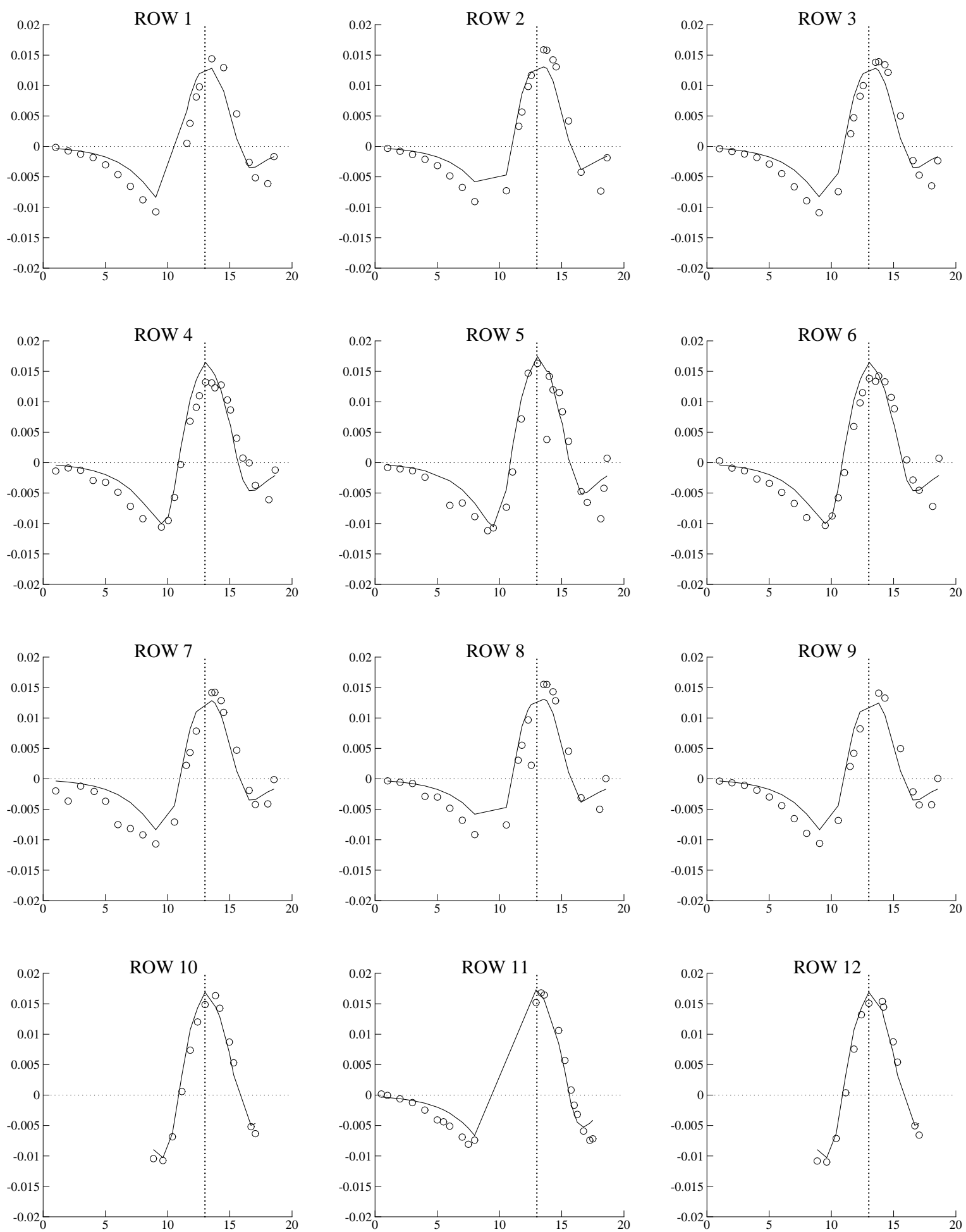

Figure 9. Comparison of wall signature, measured and calculated from TWICS $\mathrm{C}-4$ body in $2 \%$ open test section, $\mathrm{MACH}=0.95$. 

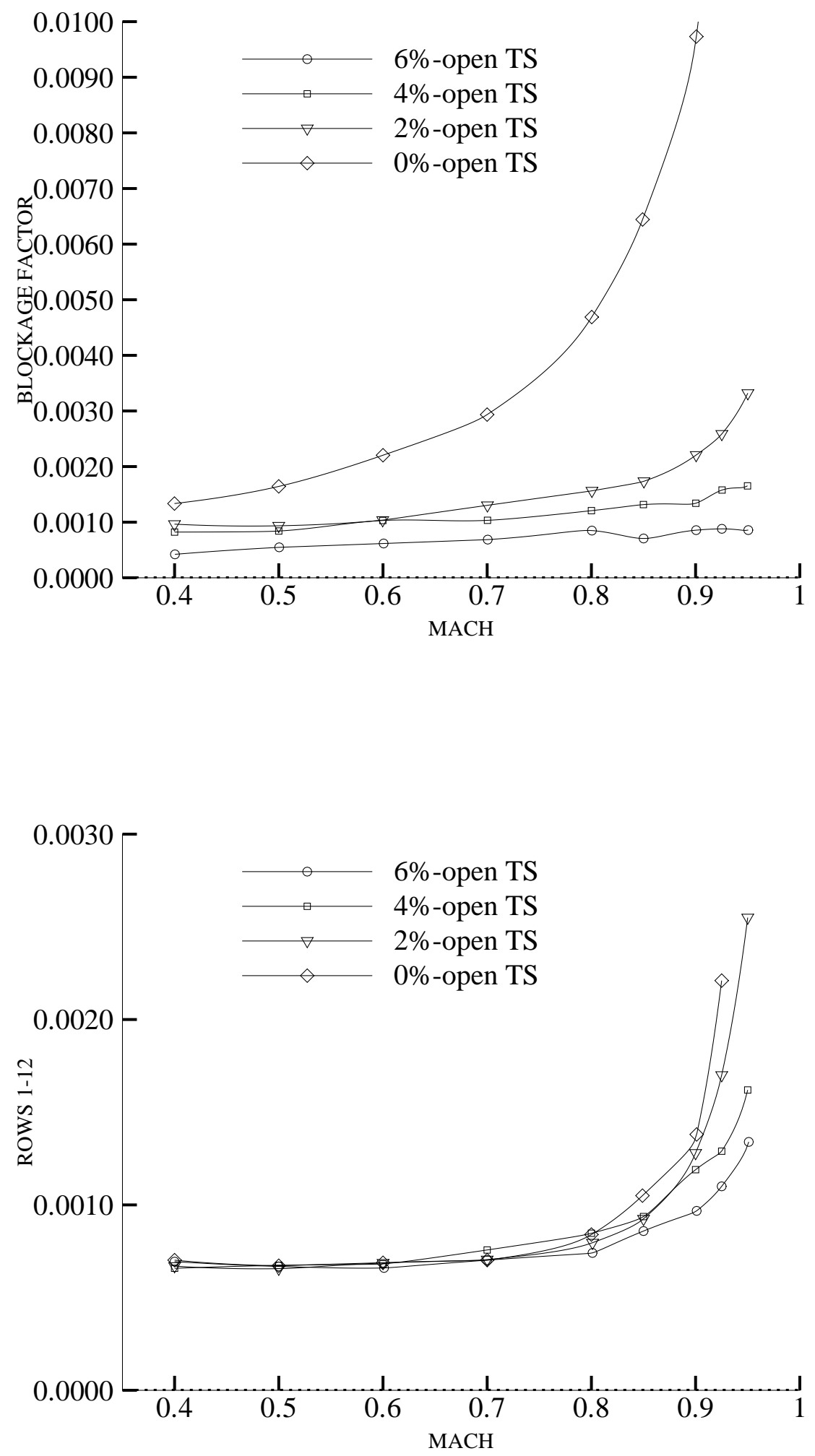

Figure 10. Blockage factor and TWICS-fit standard deviation of fit for $0 \%, 2 \%, 4 \%$ and $6 \%$. open NTF configurations, C-4 body. 

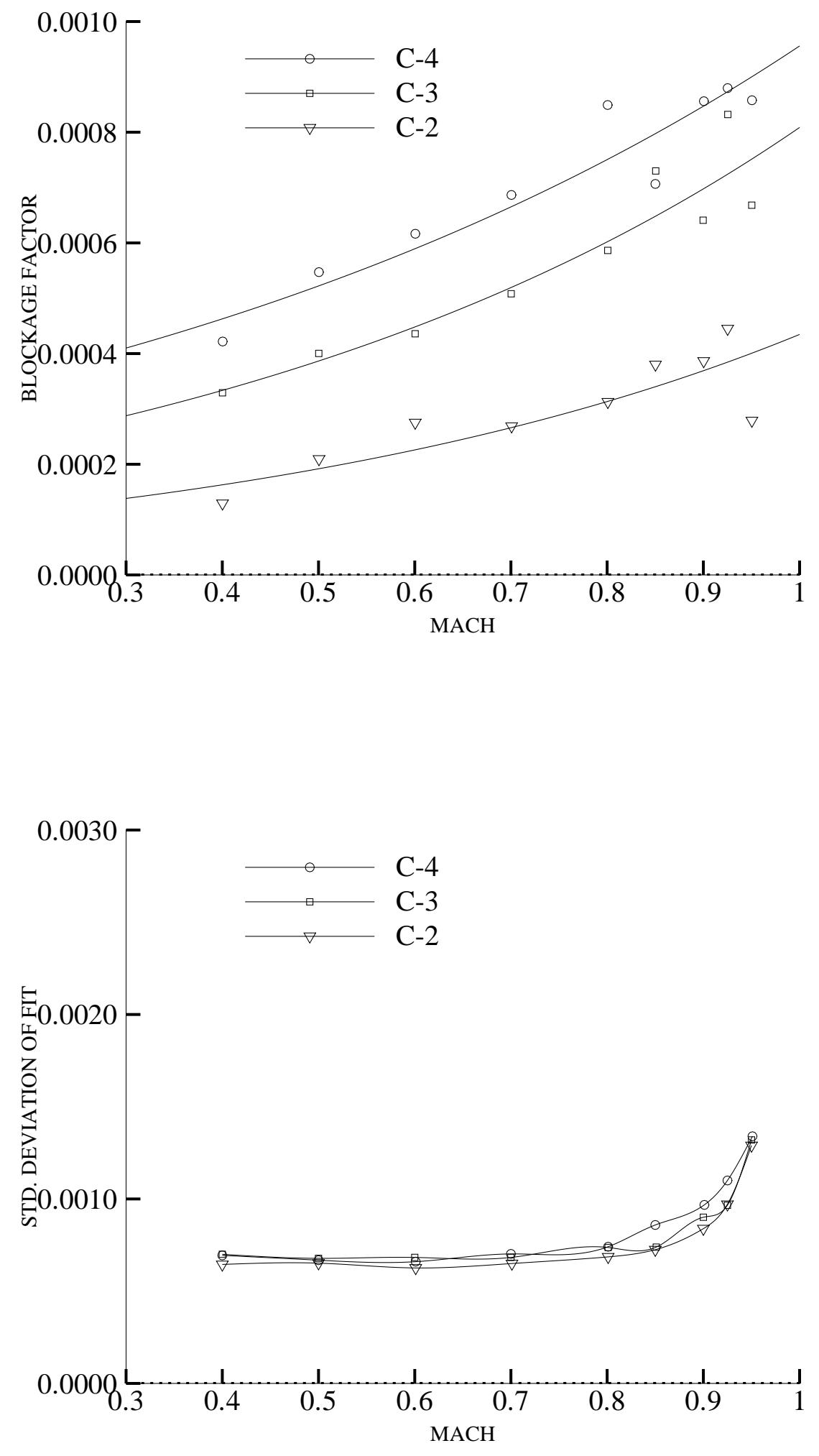

Figure 11. Blockage factor and TWICS-fit standard deviation of fit for C-4, C-3 and C-2 bodies. $6 \%$ open NTF configuration. 
Blockage parameter, e 0\%-OPEN TEST SECTION

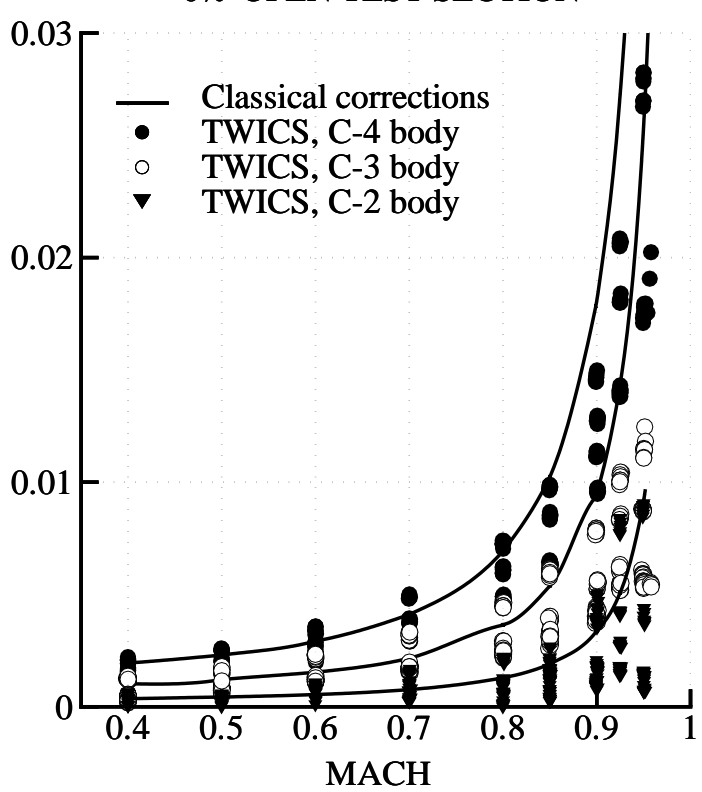

Blockage parameter, e 4\%-OPEN TEST SECTION

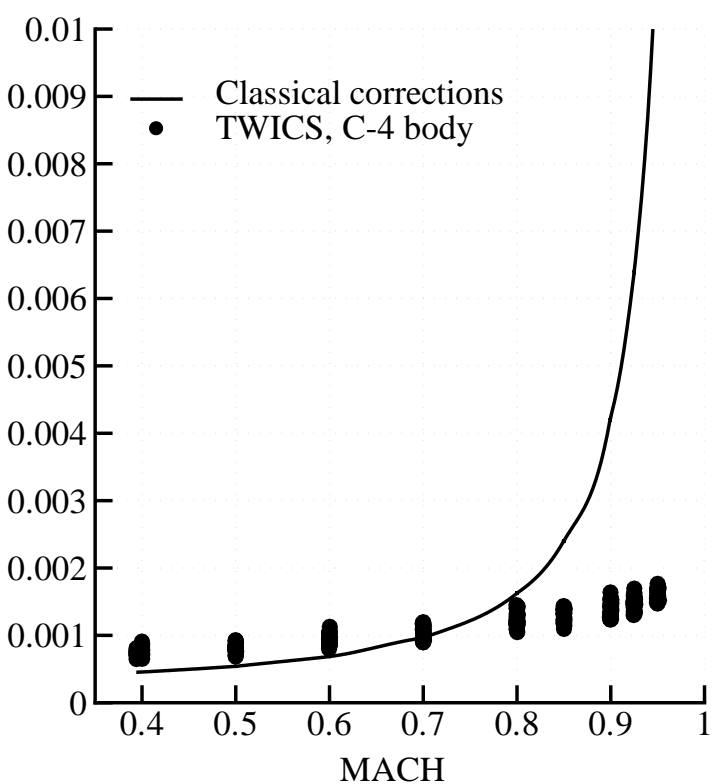

Blockage parameter, e 2\%-OPEN TEST SECTION

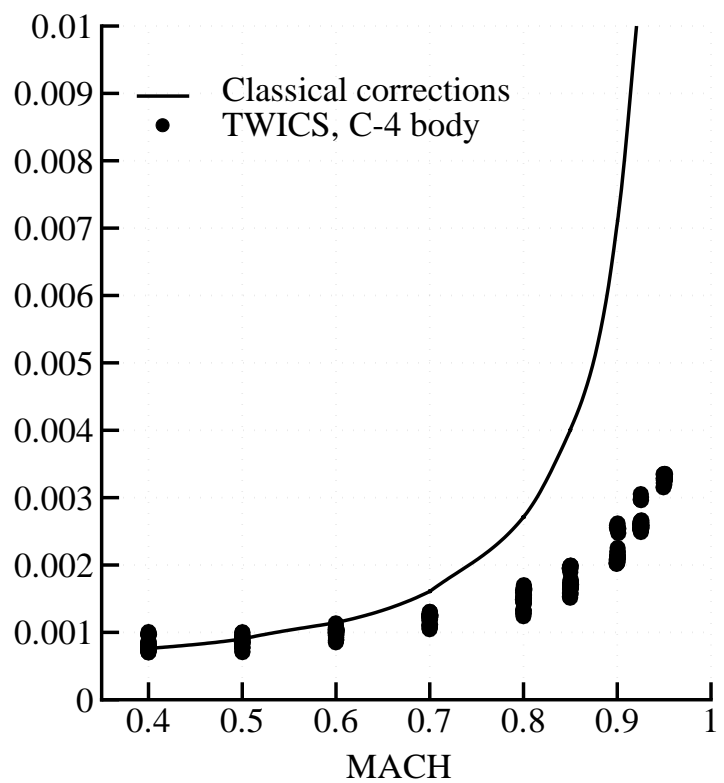

Blockage parameter, e 6\%-OPEN TEST SECTION

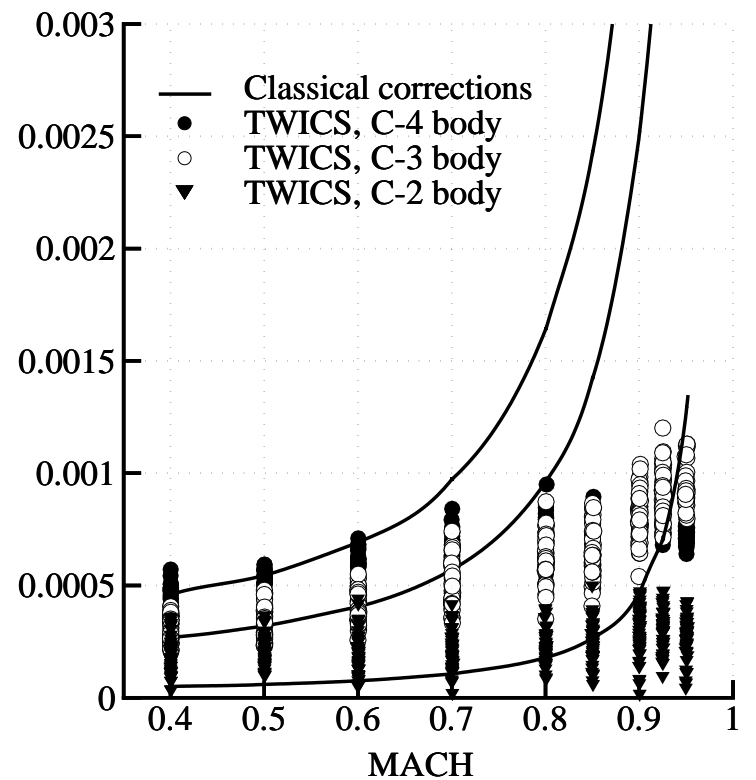

Figure 12. Comparison of classical wall correction results with TWICS wall corrections results for all test points for C-4, C-3, C-2 bodies at $6 \%, 4 \%, 2 \%$ and $0 \%$-open Test section conditions (MACH $<0.96$ points only). 